\title{
Medicina basada en la evidencia (MBE): pasado, presente y futuro
}

\author{
Evidence-based medicine (EBM): past, present and future
}

\author{
Mamani-Poma Augusto*
}

\section{Datos del Articulo \\ Universidad Privada Franz Tamayo \\ (UNIFRANZ) Sede El Alto. \\ Facultad Ciencias de la Salud. \\ Carrera de Medicina. \\ Avenida Héroes del Km 7 № 1015. \\ Esquina calle 3, Zon Villa Bolivar A. \\ Esquina calle 3, Zona Villa Bolivar A.
Tel:+591(2)2823421, +591(2)2825769, + \\ 591 (2)2823421. \\ E-mail: unifranz@unifranz.edu.bo \\ URL: http://www.unifranz.edu.bo \\ Ciudad de El Alto. La Paz. \\ Estado Plurinacional de Bolivia \\ *Dirección de contacto: \\ Universidad Privada Franz Tamayo. \\ (UNIFRANZ) Sede El Alto. \\ Avenida Héroes del Km 7 № 1015. \\ Esquina calle 3, Zona Villa Bolívar A \\ Tel: +591 (2)2823421. \\ Móvil: +591-72523100. \\ Augusto Mamani-Poma. \\ E-mail address: \\ augustomamani@gmail.com \\ Palabras clave: \\ Síntomas, \\ evidencia, \\ algoritmos.}

J. Selva Andina Res. Soc. 2018; 9(2):127-131.

\section{Historial del artículo.}

Recibido febrero, 2018.

Devuelto abril 2018

Aceptado mayo, 2018.

Disponible en línea, agosto, 2018.

Editado por:

Selva Andina

Research Society

Key words:

Symptom,

evidence,

algorithms.

\section{Sr. Editor.}

Medicina Basada en Síntomas. Pasado. La medicina en gran parte de su historia ha sido más arte que ciencia, es decir existió mucha intuición y sentido común (IlizástiguiDupuy \& Rodríguez-Rivera 2010) para poder luego tomar decisiones prácticas correctas, es por ello que se denominó Medicina Basada en Síntomas hasta los años 60 (Gracia 2009).

En el año 891-1037 un gran médico Persa Ibn Sina (Avicena), se dedicó a ver pacientes y a reunir toda la información que existía de la medicina en esa época y logró escribir un libro denominado "El Canon (Al-Qanum) de la Medicina", que fueron catorce tomos, que logró terminarse en el año 2015, que contiene la medicina islámica con muchos escritos de Galeno, Súsruta, Cháraka, además contiene la medicina Persa y China (Ferre 2003).

Luego se vino el desarrollo del pensamiento humano, la medicina como ciencia se impregnó de pensamientos filosóficos (basada en la experiencia). Los filósofos que dieron realce al método científico fue primero Francis Bacon (1561-1626), nacido en Inglaterra considerado como el padre del empirismo científico aplicando el razonamiento inductivo, además de ser considerado como uno de los pilares filosóficos de la Revolución Industrial (Toledo-Flores 2014). Luego viene René Descartes (1596-1650), nacido en Francia considerado como el padre del racionalismo deductivo y padre de la filosofía moderna, sólo la razón es la fuente del conocimiento de las verdades eternas como las matemáticas y las bases epistemológicas (Toledo-Flores 2014). Auguste Comte (1798-1857), nacido en Francia considerado como el padre del positivismo inductivo y creador de la sociología, introduce el conocimiento científico donde el conocimiento solo puede surgir de la afirmación positiva de las teorías a través del método científico, prevaleciendo ante cualquier otro pensamiento (Brennan 1999).

A la par del pensamiento filosófico se desarrolla la epistemología. Uno de los epistemólogos fue Karl Popper (1922-1994), nacido en Austria considerado como el padre del falsaciolismo deductivo o racionalismo crítico, propuso la teoría de los contrastes que si una idea existe debe ser comprobada o refutada, sin embargo ninguna idea puede 
ser comprobada al cien por ciento por lo que se genera más teorías hacia adelante (García-Jiménez 2008, González de Luna 2004). Otro gran epistemólogo fue Imre Lakatos (1922-1974), nacido en Hungría considerado como el padre del falsaciolismo sofisticado, proponiendo la teoría de los cinturones protectores de la ciencia, siendo que un problema de investigación debe ser grande y que todos los trabajos que se van realizando en el tiempo son satélites que van protegiendo el cinturón de investigación, al estudiarse todos los satélites dan como resultado una gran teoría como la teoría de la relatividad, además marcan qué rutas de investigación deben ser evitadas (heurística negativa) y cuáles seguidas (heurística positiva) (García-Jiménez 2008). Thomas Samuel Kuhn (1922-1996), nacido en Estados Unidos es considerado como el padre del paradigma científico, sostiene que toda actividad científica se desarrolla bajo un paradigma, entendido éste como el conjunto de ilustraciones recurrentes y casi normales de diversas teorías en sus aplicaciones conceptuales, instrumentales y de observación (García-Jiménez 2008).

A partir de los años 60 la medicina comenzó a convertirse en una actividad verdaderamente científica al hablar de la medicina clínica por la impregnación de pensamientos epistemológicos, más allá de la intuición y sentido común, donde Ronald Fisher (18901962) nacido en Inglaterra considerado como el padre de la estadística moderna dando origen a los métodos estadísticos y la estadística inferencial, convirtiéndose la medicina en ciencia no exacta pero muy precisa a través de la prueba de significancia estadística y de la prueba de hipótesis, siendo la estadística un punto de apoyo en innumerables áreas del conocimiento (Mateos-Aparicio 2011). Austin Bradford Hill (1897-1991) nacido en Inglaterra fue el primero en introducir el estudio clínico aleatorio de la estreptomicina en el tratamiento de la tuberculosis con el título "Streptomycin treatment of pulmonary tuberculosis a Medical Research Council investigation" publicado en British Medical Journal el 30 de octubre de 1948 (Hill 1990).

Para lograr una Medicina Basada en la Evidencia, además de la experiencia personal y el conocimiento de la patología, debe existir una integración entre una línea de pensamiento epidemiológica y bioestadística (Ortiz et al. 1998).

Medicina Basada en la Evidencia. Presente. Archibald Cochrane (1909-1988) nacido en Escocia, llega a Grecia durante la Segunda Guerra Mundial, como prisionero de guerra en Salónica y como médico calificado reflexionó que ninguno de los tratamientos que él había empleado había sido justificado por la evidencia científica de su eficacia, entonces luego de su renuncia a la medicina clínica escribió su primer artículo científico de ensayo clínico aleatorio (ECA) titulado en British Medical Journal "Sickness in Salonica: my first, worst and most successful clinical trial" de 1984 (Cochrane 1984), de tal forma que afirmaba en su libro "Effectiveness and Efficiency: Random Reflections on Health Services" de 1972, sin duda alguna es una gran crítica para nuestra profesión al no tener un resumen crítico y organizado por especialidades y subespe- 
cialidades adaptados periódicamente de todos los estudios clínicos aleatorios (Cochrane 1989). En Inglaterra lanza un reto a un grupo de médicos que no existen bases sistematizadas para la información médica, donde surge Iain Chalmers (nacido en Inglaterra 1943, edad 75 años) crea y genera el nacimiento de "The Cochrane Collaboration" fundada en 1992 que tiene por finalidad realizar resúmenes sistemáticos de los ensayos clínicos aleatorios para dar paso a lo que se conoce como metanálisis (Junquera et al. 2003).

En 1991 se origina la Medicina Basada en la Evidencia (MBE) con David Sackett (1934-2015) médico Canadiense, junto a sus colaboradores R. Brian Haynes, Gordon H. Guyatt y Peter Tugwell, de la Universidad de McMaster publican un libro titulado "Clinical Epidemiology: A Basic Science for Clinical Medicine" (Sackett et al. 1991), a partir de ello se vio la necesidad de alfabetizar metodológicamente a los médicos para una jerarquización de artículos científicos en Niveles de Evidencia y Grados de Recomendación, de tal forma que la clasificación Americana da origen a los acrónimos PICO, PECO, PIO y PEO, como una manera de identificar a los artículos de interés (Perez-Atanasio \& Sandoval-Rincón 2015).

La práctica médica siempre busca tomar sus decisiones con la mejor evidencia disponible, a menudo, esta evidencia representa extrapolaciones de principios fisiopatológicos y lógica en lugar de hechos establecidos basados en datos derivados de pacientes, es por ello que la Medicina Basada en la Evidencia (MBE) se define como el uso consciente, explícito y prudente de la mejor evidencia médica disponible para la toma de decisiones acerca de la atención médica de pacientes individuales (Sackett \& Rosenberg 1995).

Medicina Basada en Algoritmo. Futuro. Lo que está claro es que de la Medicina Basada en la Evidencia (MBE) se pasará en el futuro a la de los algoritmos, frente a la gran cantidad de datos científicos y se viene un nuevo paradigma la medicina de precisión o certeza, que consiste en poner en valor los datos genómicos de los pacientes y los relativos a su estilo de vida para dar tratamiento correcto en el momento correcto a la persona correcta, en base a las observaciones de un estudio titulado "Development and validation of a deep learning algorithm for detection of diabetic retinopathy in retinal fundus photographs" para la detección automática de la retinopatía diabética en fotografías de fondo de retina a través del desarrollo de algoritmos de alto rendimiento que proporciona una medida más confiable de la capacidad predictiva final de un modelo con el desarrollo tecnológico como es el big data (grandes conjunto de datos), siendo una nueva herramienta clínica para diagnosticar, predecir y tratar enfermedades con alta sensibilidad y especificidad, sin embargo tiene sus limitaciones la inteligencia artificial, requiriéndose más investigación para determinar la factibilidad de aplicar este 
algoritmo en el entorno clínico y para determinar si el uso del algoritmo podría conducir a una mejor atención y resultados en comparación con la evaluación oftalmológica actual (Abràmoff et al. 2013, Gulshan et al. 2016).

\section{Conclusión}

La Medicina Basada en Síntomas (pasado) fue intuitiva o puro sentido común, manifestada a través del paso histórico de la medicina.

La Medicina Basada en la Evidencia (presente) se construye en base a patrones filosóficos, epistemológicos y médicos, constituyéndose en científica y rigurosa.

La Medicina Basada en Algoritmos (futuro) pretende llegar a la precisión o la certeza diagnóstica y tratamiento, mediante la inteligencia artificial.

(C) 2018. Journal of the Selva Andina Research Society. Bolivia. All rights reserved.

\section{Conflictos de intereses}

El autor declara no tener ningún conflicto de intereses. Declaro no haber recibido ningún tipo de financiación pública y/o privada para la realización del presente manuscrito.

\section{Agradecimientos}

El autor agradece al Dr. Nelson Torrez Fiorilo de la Universidad Privada Franz Tamayo (UNIFRANZ) Sede El Alto, La Paz, Bolivia, por su aceptación para la publicación sobre Medicina basada en la evidencia (MBE): pasado, presente y futuro. De igual forma al cuerpo editorial de la Journal of the Selva Andina Research Society, Dr. Manuel Loza Murguia, por su valioso aporte a la revisión de este manuscrito.

\section{Literatura citada}

Abràmoff MD, Folk JC, Han DP, Walker JD, Williams DF, Russell SR, et al. Automated analysis of retinal images for detection of referable diabetic retinopathy. JAMA Ophthalmol 2013; 131(3):351-7.

Brennan JF. Historia y sistemas de la psicología. Pearson Educación; 1999. p. 392.

Cochrane AL. Archie Cochrane in his own words. Selections arranged from his 1972 introduction to "Effectiveness and Efficiency: Random Reflections on the Health Services" 1972. Control Clin Trials 1989;10(4):428-33.

Cochrane AL. Sickness in Salonica: my first, worst, and most successful clinical trial. Br Med $\mathrm{J}$ 1984;289(6460):1726-7.

Ferre L. Avicena Hebraico: La traducción del canon de la medicina. Sección Hebreo. Universidad de Granada: MEAH; 2003. 163-82.

García-Jiménez L. Aproximación epistemológica al concepto de ciencia: una propuesta básica a partir de Kuhn, Popper, Lakatos y Feyerabend. Andamios 2008;4(8):185-202.

González de Luna E. El concepto de sentido común en la epistemología de Karl Popper. Sig Fil 2004;6(Suppl 11):S131-144.

Gracia D. Medicina basada en la evidencia: aspectos éticos. Bioética 2009; 8(1):79-87. 
Gulshan V, Peng L, Coram M, Stumpe MC, Wu D, Narayanaswamy A, et al. Development and validation of a deep learning algorithm for detection of diabetic retinopathy in retinal fundus photographs. JAMA 2016;316(22): 2402-10.

Hill AB. Suspended judgment. Memories of the British Streptomycin Trial in Tuberculosis. The first randomized clinical trial. Control Clin Trials 1990;11(2):77-9.

Ilizástigui-Dupuy F, Rodríguez-Rivera L. El Método Clínico. Medisur. 2010;8(5):2-11.

Junquera LM, Baladrón J, Albertos JM, Olay S. Medicina basada en la evidencia (MBE): Ventajas. Rev Esp Cir Oral Maxilofac. 2003;25(5): 265-72.

Mateos-Aparicio MG. Los modelos de ecuaciones estructurales: una revisión histórica sobre sus orígenes y desarrollo. en JM. Riobóo Almanzor, I. Riobóo Lestón (Eds.), Historia de la probabilidad y de la estadística V (p. 289-301). Santiago de Compostela: AHEPE; 2011.
Ortiz Z, Garcia Dieguez M, Laffaire E. Medicina basada en la evidencia. Bol Acad Nac Med B Aires 1998;76 (2):445-54.

Pérez-Atanasio JM, Sandoval-Rincón MB. ¿Cómo formular una buena pregunta de investigación? Estructura y redacción de la pregunta de investigación. Ortho-Tips 2015;11(2):74-8.

Sackett DL, Haynes RB, Guyatt GH, Tugwell P. Clinical epidemiology: a basic science for clinical medicine, 2nd ed., Little Brown and Company, Boston; 1991. p. 441.

Sackett DL, Rosenberg WM. On the need for evidence-based medicine. J Public Health Med 1995;17(3):330-4.

Toledo-Flores LJ. Elaboración de Protocolos de Investigación; 2014. p. 89. 